\title{
Some Connections between Ground States and Temperature States of Thermodynamical Systems
}

\author{
M. SirUgue ${ }^{\star}$ and D. TeSTARD ${ }^{\star \star}$ \\ Centre de Physique Théorique, C.N.R.S., Marseille
}

Received February 16, 1970

\begin{abstract}
We investigate the properties of ground states of thermodynamical systems as limits of temperature states; we enlarge the algebra of observables in order to define a *-automorphism relevant to the study of K.M.S.-states and derive an usefull theorem of transitivity of $*$-automorphisms within the locally normal pure states of a quasilocal algebra.
\end{abstract}

\section{§ 1. Introduction}

In contrast to the situation encountered in statistical mechanics, states of field theory are generally pure states. This fact is usually explained by saying that states of field theory correspond to zero temperature and zero density.

It is our goal in this note to make this statement a little more precise and to study the behaviour of temperature states when the temperature goes to zero.

If we assume that the limits of temperature states exist in the appropriate sense, then one can derive some properties of these limits and recognize the well-known properties of a ground state; roughly speaking type I von Neumann algebras with positive hamiltonian.

We shall describe the behaviour of the states with respect to the temperature by a family of $*$-automorphisms; of course, these cannot be automorphisms of the algebra itself but merely of one of its natural enlargements. This can be achieved by extending the K.M.S. states we consider to pure states of the enlarged algebra, and by proving a theorem of transitivity of *-automorphisms within the locally normal pure states of a quasi-local algebra.

In general, we only have an existence theorem for these $*$-automorphisms and it is not sufficient to give precise results about the existence of K.M.S. states. Nevertheless, we give an example where we can construct this automorphism explicitly.

* Chargé de Recherches au C.N.R.S.

$\star \star$ Maître-Assistant - Université d'Aix-Marseille II - Centre de Luminy. 
In order to proceed, we shall briefly describe some structure we shall need in the sequel and some basic properties of the K.M.S. states.

Some results we shall give are independent of the nature of the algebra we shall choose; nevertheless in view of physical applications and due to the main theorem of Section 4; we shall be mainly interested in the quasi-local algebra or in algebras having essentially the same features; more precisely:

Definition 1.1. $A C^{*}$-algebra $\mathscr{A}$ has a quasi-local structure if

$\mathrm{i}-\mathscr{A}$ has a type $I_{\infty}$ sequential funnel, i.e.: there exists an increasing sequence $\left\{\mathscr{M}_{n}\right\}_{n=1 \ldots n \ldots}$ of type $I_{\infty}$ factors with at least one faithful representation $\Phi_{n}$ in a separable Hilbert space.

ii - for every $n$ there exists $n^{\prime}>n$ such that the commutant of $\mathscr{M}_{n}$ in $\mathscr{M}_{n^{\prime}}$ is infinite.

iii- $\mathscr{A}=\bar{\bigcup}_{n} \mathscr{M}_{n} \|$

we refer to [1] and [2] for the physical motivations of this choice and for more details.

Definition 1.2. A state (resp. a representation) of $\mathscr{A}$ is locally normal whenever it is normal in restriction to each $\mathscr{M}_{n}$.

Notice that a locally normal state induces a locally normal cyclic representation of $\mathscr{A}$ (and conversely) ([2], prop. 6) and that these representations act within separable Hilbert space ([2], prop. 8); moreover the algebra is simple ([2], prop. 10).

Let us remark that if $R$ is a locally normal representation of $\mathscr{A}$ in $H_{1}, R_{n}$ its restriction to $\mathscr{M}_{n}$ then (up to unitary equivalence), one has:

$$
H_{1}=H \otimes H
$$

where $H$ is the standard separable Hilbert space, and

$$
R_{n}(A)=\Phi_{n}(A) \otimes I \quad A \in \mathscr{M}_{n} .
$$

- We shall need in the sequel the opposite algebra $\mathscr{A}^{v}$ of a normed *-algebra $\mathscr{A}$; by definition there exists a linear isometric bijection $i$ from $\mathscr{A}$ onto $\mathscr{A}^{v}$ such that

$$
\begin{aligned}
i(A) i(B) & =i(B A) \quad \forall A, B \in \mathscr{A} \\
i(A)^{*} & =i\left(A^{*}\right)
\end{aligned}
$$

so that if $\mathscr{A}$ is a $C^{*}$-algebra, $\mathscr{A}^{v}$ is a $C^{*}$-algebra too.

- If $S$ is an antirepresentation of $\mathscr{A}$ it can be turned into a representation $S^{v}=S \circ i^{-1}$ of $\mathscr{A}^{v}$.

- If now $H$ is an Hilbert space we can define the Hilbert space $H^{v}$ as follows: by definition there exists an antiunitary operator $\mathscr{V}$ from 
$H$ onto $H^{v}$ so that if $\pi$ is a representation of $\mathscr{A}$ in the Hilbert space $H, \pi^{*}$ defined through:

$$
\pi^{*}(i(A))=\mathscr{V} \pi\left(A^{*}\right) \mathscr{V}^{-1}
$$

is a representation of $\mathscr{A}^{v}$ in $H^{v}$; this correspondence preserves irreducibility, factoriality and faithfulness.

- If $\mathscr{A}$ has a quasi-local structure given by the funnel $\left\{\mathscr{M}_{n}\right\}$ then obviously $\mathscr{A}^{v}$ has a quasi-local structure given by the funnel $\left\{\mathscr{M}_{n}^{v}\right\}$ ( $\Phi_{n}^{*}$ is a faithful representation of $\mathscr{M}_{n}^{v}$ in a separable Hilbert space).

- Analogously $\mathscr{A} \otimes \mathscr{A}^{v}$ (as defined e.g. in [3], chap. III, def. 2.1) has a quasi-local structure if we define its funnel to be $\left\{\mathscr{M}_{n} \otimes \mathscr{M}_{n}^{v}\right\}$.

Finally let us quote the essential features of the K.M.S. states (see definition below) of a quasi-local algebra. We refer to $[1,4]$ for a complete derivation of these results and for further details.

Definition 1.3. Let $t \rightarrow \alpha_{t}$ a weakly continuous homomorphism of the additive group of reals into the $*$-automorphism group of a $C^{*}$-algebra $\mathscr{A}$ (an evolution of $\mathscr{A})$. A state $\omega_{\beta}$ of $\mathscr{A}$ is said to be a K.M.S. state with respect to $\alpha_{t}$ at the inverse temperature $\beta$ if for any function $f$ with Fourier transform in $\mathscr{D}$, one has:

$$
\int f(t-i \beta) \omega_{\beta}\left(A \alpha_{t} B\right) d t=\int f(t) \omega_{\beta}\left(\alpha_{t} B . A\right) d t
$$

for any $A$ and $B$ in $\mathscr{A}$.

- We list now the salient features of a K.M.S. state $\omega_{\beta}$ (see e.g. $[1,4,5])$.

$\mathrm{i}-\omega_{\beta}$ is $\alpha_{t}$-invariant; so that $\alpha_{t}$ is unitarily implemented by a strongly continuous unitary group

such that:

$$
t \rightarrow U_{\beta}(t)
$$

$$
\text { ii }-U_{\beta}(t) \Omega_{\beta}=\Omega_{\beta}
$$

where $\Omega_{\beta}$ is the cyclic vector associated to $\omega_{\beta}$.

iii - The spectrum of the infinitesimal generator of $U_{\beta}(t)$ is a subset of the real line symmetric with respect to the origin.

$$
\text { iv - } U_{\beta}(t) \notin R_{\beta}(\mathscr{A})^{\prime \prime} \quad U_{\beta}(t) \notin R_{\beta}(\mathscr{A})^{\prime}
$$

where $R_{\beta}$ is the representation of $\mathscr{A}$ constructed from $\omega_{\beta}$.

$\mathrm{v}$ - If has a quasi-local structure, then $R_{\beta}$ acts within a separable Hilbert space.

vi- Associated with $R_{\beta}$ there exists an antirepresentation $S_{\beta}$ of $\mathscr{A}$ such that

$$
\left\{R_{\beta}(\mathscr{A})\right\}^{\prime \prime}=\left\{S_{\beta}(\mathscr{A})\right\}^{\prime}
$$

(in [5] one considers an antilinear representation of $\mathscr{A}$ ).

vii $-\Omega_{\beta}$ is cyclic and separating for $R_{\beta}$ (and consequently for $S_{\beta}$ ). 


\section{$\S$ 2. The Limiting Case $\beta=\infty$; Properties of the Ground State}

We uncritically adopt here the point of view that the K.M.S. boundary condition defines the temperature state of the system. This may exclude phase transitions (cf. [5]) at least of certain type. Nevertheless this condition is meaningless for $T=0$. If there exists no phase transition at this point, we can only expect to obtain the corresponding state through a limiting process.

In such a general situation we are not able to ensure the existence of a ground state i.e. a limit for $\beta \rightarrow \infty$ of a family of K.M.S. states $\omega_{\beta}$, so we shall assume this limit exists pointwise viz in the $w^{*}$-topology sense.

Furthermore the previous formulation of the K.M.S. condition is not appropriate for this limiting process and we shall derive another equivalent formulation.

Lemma 2.1. With the same notation as previously, the following are equivalent

$\mathrm{i}-\omega_{\beta}$ is a K.M.S. state at the inverse temperature $\beta$

ii - for every function $\hat{f}$, with Fourier transform $f$ in $\mathscr{D}$ and for any pair of elements $A, B$ in $\mathscr{A}$

$$
\begin{aligned}
\int_{-\infty}^{+\infty} d t \hat{f}(t) \omega_{\beta}\left(A \alpha_{t} B-\alpha_{t} B . A\right) \\
=\int_{-\infty}^{+\infty} d t \widehat{\hat{f h \pi} \beta}(t) \omega_{\beta}\left(A \alpha_{t} B+\alpha_{t} B . A\right)
\end{aligned}
$$

where $f \operatorname{th} \pi \beta(\xi)=f(\xi) \operatorname{th}(\pi \beta \xi) \quad f \in \mathscr{D}$.

Proof. Notice that $e_{\beta}+1: \xi \rightarrow e^{2 \pi \beta \xi}+1$ is a regular factor (cf. e.g. [6]) so that $\mathrm{ii}-$ is equivalent to

$$
\int_{-\infty}^{+\infty} \overline{f\left(e_{\beta}+1\right)}(t) \omega_{\beta}\left(\left[A, \alpha_{t} B\right]_{-}\right) d t=\int_{-\infty}^{+\infty} \overline{f\left(e_{\beta}-1\right)}(t) \omega_{\beta}\left(\left[A, \alpha_{t} B\right]_{+}\right) d t .
$$

A direct calculation shows that (2.3) is equivalent to:

$$
\int_{-\infty}^{+\infty} \widehat{f e_{\beta}}(t) \omega_{\beta}\left(A \alpha_{t} B\right) d t=\int_{-\infty}^{+\infty} \hat{f}(t) \omega_{\beta}\left(\alpha_{t} B \cdot A\right) d t
$$

which completes the proof.

The previous lemma gives a straightforward proof of the pointwise invariance of the center of $\pi_{\beta}(\mathscr{A})^{\prime \prime}$ if one notices that the K.M.S. boundary condition extends to the continuous extension of $\omega_{\beta}$ to $\pi_{\beta}(\mathscr{A})^{\prime \prime}$.

Now we shall study the properties of a special type of states which will be relevant in the sequel: 
Definition 2.4. Let $t \rightarrow \alpha_{t}$ be an evolution of $\mathscr{A}$; a state $\varrho_{\infty}$ of $\mathscr{A}$ is said to be of the Fock type with respect to $\alpha_{t}$ iff

$$
\int_{-\infty}^{+\infty} f(t) \varrho_{\infty}\left(A \alpha_{t} B\right) d t=0
$$

for any $A$ and $B$ in $\mathscr{A}$ and every $f$ with Fourier transform $\hat{f}$ in $\mathscr{D}$ and supp. $\hat{f}()-\infty, 0($.

Such states have very interesting properties, quite different of those of the K.M.S. state. Most of them are well known from usual field theory and for sake of completeness we shall rederive them in order to compare the situation here to the one encountered for temperature states.

Lemma 2.5. Let $\varrho_{\infty}$ be a Fock type state; then it is an invariant state.

Proof. From the very definition of Fock type state one has equivalently

$$
\int_{-\infty}^{+\infty} f(t) \varrho_{\infty}\left(\alpha_{t} A . B\right) d t=0
$$

for $f$ with Fourier transform $\hat{f}$ in $\mathscr{D}$ and Supp $\hat{f} C) 0+\infty$ (and for any $A$ and $B$ in the algebra; consider now

$$
t \in \mathbb{R} \rightarrow \varrho_{\infty}\left(\alpha_{t} A\right)
$$

it is a continuous and bounded function; hence it is the Fourier transform of a distribution.

Let now $f$ be a function the Fourier transform $\hat{f}$ of which is in $\mathscr{D}$ and such that Supp $\hat{f} \cap\{0\}=\emptyset$; then $f=f^{+}+f^{-}$with supp $\left.\overrightarrow{f^{+}} C\right) 0, \infty($ and supp $\left.\overrightarrow{f^{-}} C\right)-\infty, 0($.

From the previous remark,

$$
0=\int_{-\infty}^{+\infty} f(t) \varrho_{\infty}\left(\alpha_{t} A\right) d t=\int_{-\infty}^{+\infty} d t f^{+}(t) \varrho_{\infty}\left(\alpha_{t} A\right)+\int_{-\infty}^{+\infty} d t f^{-}(t) \varrho_{\infty}\left(\alpha_{t} A\right)
$$

hence $\varrho_{\infty}\left(\alpha_{t} A\right)$ is the Fourier transform of a distribution with support on $\{0\}$ consequently, by standard boundedness arguments, it is a constant.

Lemma 2.6. Let $\varrho$ be a state of a $C^{*}$-algebra $\mathscr{A}$, let $\pi_{\varrho}$ be the representation in $\mathscr{H}_{\varrho}$ with cyclic vector $\Omega_{\varrho}$ defined by $\varrho$. Assume there exists a group of unitaries $U_{\varrho}(t)$ which implement the evolution $\alpha_{t}$ of $\mathscr{A}$ and let $H_{\varrho}$ the corresponding infinitesimal generator; the following are equivalent

$\mathrm{i}-\varrho_{\infty}$ is of Fock type with respect to $\alpha_{t}$

ii- $H_{\varrho} \geqq 0$ and $H_{\varrho} \Omega_{\varrho}=0$.

Proof. For every $\psi$ in $\mathscr{H}_{\varrho}$

$$
t \rightarrow\left(\psi \mid U_{\varrho}(t) \psi\right)
$$


is a function of positive type; let $\mu_{\psi}$ be the corresponding positive measure. Consider now the set of vectors defined by the Bochner integral:

$$
\psi_{A f}=\int_{-\infty}^{+\infty} d t f(t) U_{\varrho}(t) A \Omega_{\varrho} \quad A \in \pi_{\varrho}(\mathscr{A}) .
$$

One has $(\forall f \in \mathscr{D})$

$$
\left.\left(\psi_{A f} \mid H_{\varrho} \psi_{A f}\right)=\mu_{A f}(\omega)|f|^{2}\right) \quad(\omega g)(\omega)=\omega g(\omega)
$$

the equivalence follows if one notices that the $\psi_{A f}$ 's are dense in $\mathscr{H}_{Q}$.

In contrast to the non-zero temperature states case, the spectrum of the Hamiltonian $H_{e}$ is completely unsymmetrical.

Lemma 2.7. (cf. [7]) Let $\pi_{\varrho}(\mathscr{A})^{\prime}$ the commutant of $\pi_{\varrho}(\mathscr{A}), \pi_{\varrho}(\mathscr{A})^{\prime}$ $C\left\{U_{\varrho}(t) ; t \in R\right\}^{\prime}$ or equivalently $H_{\varrho}$ is affiliated to $\pi_{\varrho}(\mathscr{A})^{\prime \prime}$.

Proof. Let us consider the continuous and bounded function

$$
t \rightarrow\left(\Omega_{\varrho} \mid A^{*} U_{\varrho}(t) B \Omega_{\varrho}\right) \quad A^{*} \in \pi_{\varrho}(\mathscr{A})
$$

and $B$ in $\pi_{\varrho}(\mathscr{A})^{\prime}$; one shows by an argument quite similar to the one used in Lemma 2.5 that it is the Fourier transform of a distribution with support on $\{0\}$, hence a constant; using the cyclicity of $\Omega_{\varrho}$ with respect to $\pi_{\varrho}(\mathscr{A})^{\prime \prime}$ and its separating character with respect to $\pi_{\varrho}(\mathscr{A})^{\prime}$ one gets the result. In order to proceed we need some assumption on the evolution since essentially one has to exclude the trivial case where the evolution is trivial on a subalgebra and consequently the K.M.S. state over this subalgebra is a central state whatever be the temperature.

Lemma 2.9. (cf. [7]). Let $\mathscr{A}$ be $\alpha_{t}$ abelian in the sense of [8], then $\pi_{\varrho}(\mathscr{A})^{\prime}$ is abelian and consequently $\pi_{\varrho}(\mathscr{A})^{\prime \prime}$ is of type $I$.

The proof is immediate.

In contrast to the case of temperature states, the commutant $\pi_{\varrho}(\mathscr{A})^{\prime}$ is no longer isomorphic to $\pi_{\varrho}(\mathscr{A})^{\prime \prime}$ in fact $\Omega_{\varrho}$ is not a separating vector for $\pi_{\varrho}(\mathscr{A})^{\prime \prime}$.

We come now to the main result of this section in order to show the interest of the previously defined states (cf. $[9,10,16])$.

Proposition 2.10. Let $\alpha_{t}$ be an evolution of the $C^{*}$-algebra $\mathscr{A}$. Assume that there exists a solution $\varrho_{\beta}$ of the K.M.S. boundary condition (def. 1.3) for every $\beta>\beta_{0}$ and that:

$$
\varrho_{\beta} \underset{\beta \rightarrow \infty}{\longrightarrow} \varrho_{\infty}
$$

where the convergence is in $w^{*}$-topology; then $\varrho_{\infty}$ is of Fock type. 
Proof. (cf. [9]). Consider $\hat{f} \in \mathscr{D} \operatorname{Supp} \hat{f} \subset)-\infty, 0$ ( then by (2.3)

$$
\begin{aligned}
& \int_{-\infty}^{+\infty} d t \overline{f(t h \pi \beta)} \varrho_{\beta}\left(\left[A, \alpha_{t} B\right]_{+}\right)-\int_{-\infty}^{+\infty} d t \hat{f}(t) \varrho_{\infty}\left(\left[A, \alpha_{t} B\right]_{+}\right) \\
& =\int_{-\infty}^{+\infty} d t \overline{f(t h \pi \beta-1)}(t) \varrho_{\beta}\left(\left[A, \alpha_{t} B\right]_{+}\right)+\int d t \hat{f}(t)\left(\varrho_{\beta}-\varrho_{\infty}\right)\left(\left[A, \alpha_{t} B\right]_{+}\right) .
\end{aligned}
$$

Moreover both terms go to zero as $\beta \rightarrow \infty$, the second one due to the assumed convergence of $\varrho_{\beta}$ 's. For the first one $|t h \pi \beta-1|<1$ for $\xi>0$ so that one can twice exchange the limit $\beta \rightarrow \infty$ with the integration; moreover pointwise

$$
\lim _{\beta \rightarrow \infty} t h(\pi \beta \xi)-1=0 \text { for } \xi>0
$$

and $\varrho_{\beta}\left(\left[A, \alpha_{t} B\right]_{+}\right)$is bounded independently of $\beta$.

\section{§3. The Purification Process}

In this section, we shall generalize a construction due to Powers and Størmer in the special case of quasi-free states of Clifford algebra [11]. Indeed quasi-free states with cyclic and separating vectors are K.M.S. states with respect to a suitable evolution (see e.g. [9]). They show that, given a quasi-free state $\omega$, there exists an extension of $\omega$ which is a pure quasi-free state of an enlarged Clifford algebra. It is then natural to ask whether this is generally true.

For the sake of clarity, we shall perform this construction for a temperature state of a system of particles enclosed within a finite volume $\mathrm{V}$.

The algebra is $\mathscr{B}\left(\mathscr{H}_{V}\right)$ where $\mathscr{H}_{V}$ is the Fock space associated with V; the equilibrium state at the inverse temperature $\beta$ is given by the density matrix.

$$
\left.\varrho_{\beta}=\exp \left(-\beta H_{V}\right)=\sum_{i} e^{-\beta_{\varepsilon i}} \mid \varphi_{i}\right)\left(\varphi_{i} \mid\right.
$$

$H_{V}$ is the hamiltonian of the system (including the chemical potential see [5]).

The standart realization of the associated representation can be described as follows:

$k_{0}=\sqrt{\varrho_{\beta}}$ is a Hilbert Schmidt (H.S.) operator hence the representation space can be considered as the Hilbert space of (H.S.) operators. $k_{0}$ is a cyclic vector and

$$
R_{\beta}(A) k_{0}=A k_{0} \in \text { H.S. } \quad A \in \mathscr{B}\left(\mathscr{H}_{v}\right)
$$


furthermore one has within the same space with the same cyclic vector a representation of $\mathscr{A}^{v}$

$$
S_{\beta}(i(A))=k_{0} A .
$$

On the other hand using the natural isomorphism between the (H.S.) operator space and the tensor product $\mathscr{H}_{V} \otimes \mathscr{H}_{V}^{v}$

$$
\left.\sum_{n, m} \lambda_{n m} \mid \varphi_{n}\right)\left(\varphi_{m} \mid \rightarrow \sum_{n, m} \lambda_{n m} \varphi_{n} \otimes \mathscr{V} \varphi_{m} \quad \varphi_{n}, \varphi_{m} \in \mathscr{H}_{V}\right.
$$

we get for the representation $R_{\beta}$

$$
R_{\beta}(A)=A \otimes I .
$$

Analogously

$$
S_{\beta}(i(A))=I \otimes A
$$

which exhibits explicitly the commutation theorem.

If we now consider the algebra $\mathscr{A} \otimes \mathscr{A}^{v}$ in $\mathscr{H}_{V} \otimes \mathscr{H}_{V}^{v}$ then

$$
\tilde{R}_{\beta}(A \otimes i(B))=R_{\beta}(A) S_{\beta}(i(B))
$$

which is the identity representation is irreducible and reduces to the previous one for elements of the form $A \otimes 1$.

Consider now the two vector states of this representation defined by the vectors:

$$
\begin{aligned}
& \tilde{\Omega}_{F}=\Omega_{F} \otimes v \Omega_{F} \\
& \tilde{\Omega}_{\beta}=\sum_{i}^{\infty} e^{\frac{-\beta \varepsilon_{i}}{2}} \varphi_{i} \otimes v \varphi_{i} .
\end{aligned}
$$

There exists an isomorphism $\alpha_{\beta}$ (actually a spatial isomorphism of $\mathscr{A} \otimes \mathscr{A}^{v}=\mathscr{B}\left(\mathscr{H}_{V}\right) \otimes \mathscr{B}\left(\mathscr{H}_{V}^{v}\right)$ such that

$$
\omega_{\tilde{\Omega}_{\beta}}=\omega_{\tilde{\Omega}_{F}} \circ \alpha_{\beta} .
$$

Another example of this situation is given by quasi-free states of the CCR algebra $\overline{\Delta(H, \sigma)}$ defined in [12]. Suppose that the one-particle space is $\sigma$-complete (cf. [13], p. 295); a representation $\pi$ given by a quasi-free state corresponding to the operator $A_{s}$ can be written:

$$
\pi\left(\delta_{\varphi}\right)=\pi_{J}\left(\delta_{T+\varphi}\right) \otimes \pi_{-J}\left(i\left(\delta_{T-\varphi}\right)\right)
$$

where $\pi_{J}$ (resp. $\pi_{-J}$ ) is the representation of $\overline{\Delta(H, \sigma)}$ (resp. $\overline{\Delta(H, \sigma)}{ }^{v}$ $=\overline{\Delta(H,-\sigma)})$ associated to the complex structure $J$, the polar part of $A_{s}$ and $T_{ \pm}=\frac{1}{\sqrt{2}}\left(\left|A_{s}\right| \pm 1\right)^{\frac{1}{2}}$. Within the same Hilbert space there is an 
antirepresentation $\pi^{\prime}$ given by

$$
\pi^{\prime}\left(\delta_{\varphi}\right)=\pi_{J}\left(\delta_{T-\varphi}\right) \otimes \pi_{-J}\left(i\left(\delta_{T+\varphi}\right)\right) .
$$

Consider now the representation $\tilde{\pi}$ of

$$
\overline{\Delta(H, \sigma)} \otimes \overline{\Delta(H, \sigma)^{v}}=\overline{\Delta(H, \sigma)} \otimes \overline{\Delta(H,-\sigma)}=\overline{\Delta(H \oplus H, \sigma \oplus(-\sigma))}
$$

given by

$$
\tilde{\pi}\left(\delta_{\varphi} \otimes i\left(\delta_{\psi}\right)\right)=\pi\left(\delta_{\varphi}\right) \pi^{\prime}\left(\delta_{\psi}\right)=\pi_{J}\left(\delta_{T+\varphi+T-\psi}\right) \otimes \pi_{-J}\left(i\left(\delta_{T-\varphi+T+\psi}\right)\right)
$$

It is obtained from the representation $\pi_{J} \otimes \pi_{-J}$ by the Bogoliubov transformation of $\overline{\Delta(H \oplus H, \sigma \oplus(-\sigma))}$

$$
T=\left[\begin{array}{cc}
T_{+} & T_{-} \\
T_{-} & T_{+}
\end{array}\right]
$$

Consider now the physically interesting case where $\mathscr{A}$ is a quasi-local algebra. Let $\omega_{\beta}$ be a factor K.M.S. (hence locally normal) state. Let be $R_{\beta}$ and $S_{\beta}$ the representations previously defined. Their restriction to $\mathscr{M}_{n}$ can be written as:

$$
\begin{aligned}
R_{\beta}(A) & =\Phi_{n}(A) \otimes I \otimes I \quad A, B \in \mathscr{M}_{n} \\
S_{\beta}(i(B)) & =I \otimes \Phi_{n}^{*}(B) \otimes I
\end{aligned}
$$

hence

$$
\tilde{\pi}: A \otimes i(B) \rightarrow R_{\beta}(A) S_{\beta}(B)=\Phi_{n}(A) \otimes \Phi_{n}^{*}(B) \otimes I
$$

defines a representation of $\mathscr{M}_{n} \otimes \mathscr{M}_{n}^{v}$. It extends to the norm closure of $\bigcup_{n} \mathscr{M}_{n} \otimes \mathscr{M}_{n}^{v}$. Moreover $\tilde{\pi}$ is irreducible since $R_{\beta}$ is a factor representation and $\left\{R_{\beta}(\mathscr{A})\right\}^{\prime}=\left\{S_{\beta}(\mathscr{A})\right\}^{\prime \prime}$.

Let us remark that this representation is locally normal by construction.

Let us consider now a locally normal irreducible representation $\pi_{F}$ of $\mathscr{A}$ (for instance associated to the ground state of the system if it exists) then $\tilde{\pi}_{F}=\pi_{F} \otimes \pi_{F}^{*}$ defines another irreducible representation of $\mathscr{A} \otimes \mathscr{A}^{v}$; the question is now whether there exists a $*$-automorphism of $\mathscr{A} \otimes \mathscr{A}^{v}$ which connects this representation to the previous one; an affirmative answer is given in the next section.

\section{§ 4. A Theorem of Transitivity}

Above discussion has shown the interest of the transitivity of automorphisms within a particular class of pure states of the algebra of observables. This transitivity exists at least in the two examples of 
previous section, and we shall obtain a similar result for locally normal pure states of the quasi-local algebra of observables.

Let us give first some preliminary results:

Lemma 4.1. Let $\mathscr{A}$ be a $C *$-algebra with unit acting on a separable Hilbert space $H$; let $F=\left\{\mathscr{M}_{\alpha}\right\}$ be a net in $\mathscr{A}$ (cf. [2]); let $U$ be a unitary operator of the von Neumann algebra $\mathscr{A}^{\prime \prime}$ generated by $\mathscr{A}$ then:

for any $\varepsilon>0$ and finite set $\left\{f_{j}\right\}_{j=1 \ldots n}$ of vectors in $H$ there exists an $\alpha$ and a unitary operator $V$, element of $\mathscr{M}_{\alpha}$, such that

$$
\left\|(U-V) f_{j}\right\|<\varepsilon \quad \forall j=1 \ldots n .
$$

Proof. Let $A$ be self-adjoint in $\mathscr{A}^{\prime \prime}$ chosen such that

$$
\begin{gathered}
\|A\| \leqq 1 \\
U=\exp (i \pi A)
\end{gathered}
$$

then by strong continuity of $A \rightarrow \exp (i \pi A)$ on the unit ball of $\mathscr{B}(H)$ and Kaplansky density theorem (cf. [14], p. 43, th. 3), there exists a selfadjoint $B$ of norm less or than equal to one in $\bigcup_{\beta} \mathscr{M}_{\beta}$ such that

$$
\left\|(\exp (i \pi A)-\exp (i \pi B)) f_{j}\right\| \leqq \varepsilon \quad \forall j=1 \ldots n .
$$

Moreover $V=\exp (i \pi B)$ is in $\bigcup_{\beta} \mathscr{M}_{\beta}$; indeed each $\mathscr{M}_{\beta}$ is norm closed,

Lemma 4.2. Let $\mathscr{R}, \mathscr{R}_{1}$ and $\mathscr{R}_{2}$ be factors of type $I_{\infty}$ on a separable Hilbert space, such that $\mathscr{R}_{1}$ and $\mathscr{R}_{2}$ are contained in $\mathscr{R}$ in which they have an infinite relative commutant. Then there exists an unitary operator $U$ in $\mathscr{R}$ such that

$$
U \mathscr{R}_{1} U^{-1}=\mathscr{R}_{2} .
$$

Proof. There exists an isomorphism of $\mathscr{R}$ onto $\mathscr{B}(H) \otimes \mathbb{1}_{K}$ where $H$ is a separable Hilbert space (cf. [14], p. 124, corr. 3), consequently we can assume $\mathscr{R}$ to be $\mathscr{B}(H)$. Now let $U_{i}(i=1,2)$ be an isomorphism of $H$ onto $H \otimes H$ such that the corresponding spatial isomorphism sends $\mathscr{R}_{i}$ onto $\mathscr{B}(H) \otimes \mathbb{1}$ and $\mathscr{R}_{i}^{\prime}$ onto $\mathbb{1} \otimes \mathscr{B}(H)$; now we can choose $U=U_{2}^{-1} U_{1}$.

Up to now all the algebras we consider have a quasi-local structure in the sense of Definition 1.1.

Lemma 4.3, Let $\mathscr{A}=\bar{\bigcup}_{n} \mathscr{M}_{n}$ and $\mathscr{B}=\bar{\bigcup}_{m} \mathscr{N}_{m}$ be $C *$-algebras with a quasi-local structure acting on a separable Hilbert space $H$ and generating the same type $I_{\infty}$ factor $\mathscr{R}$. Let $\Omega$ be a strong neighbourhood of the identity in $\mathscr{R}$. For any $n$, there exists $m \geqq n$, a factor $\mathscr{N}$ of type $I_{\infty}$ con- 
tained in $\mathcal{N}_{m}$ and a unitary operator $U$ in $\Omega$ such that:

$$
U \mathscr{M}_{n} U^{-1}=\mathscr{N} .
$$

Proof. By Lemma 4.2, there exists a unitary operator $U_{1}$ in $\mathscr{R}$ such that:

$$
U_{1} \mathscr{M}_{n} U_{1}^{-1}=\mathscr{N}_{n} .
$$

Choosing, by Lemma 4.1, $U_{2}$ in $\mathscr{N}_{n^{\prime}}$ such that

$$
U_{1} \cdot U_{2}^{-1} \in \Omega
$$

we can put $m=\sup \left(n, n^{\prime}\right)$ and $U=U_{2} U_{1}$, then

$$
\mathscr{N}=U_{2} \mathscr{N}_{n} U_{2}^{-1} \subset \mathscr{N}_{m} .
$$

Lemma 4.4. Let $\mathscr{A}=\overline{\bigcup_{n} \mathscr{M}_{n}}, \mathscr{B}=\bar{\bigcup}_{m} \mathscr{N}_{m}$ be $C *$-algebras with a quasilocal structure acting on a separable Hilbert space $H$ and generating the same factor $\mathscr{R}$ of type $I_{\infty}$. Let $\mathscr{N}$ be a type $I_{\infty}$ factor in $\mathscr{N}_{m}$; assume there exists an unitary operator of $\mathscr{R}$ such that

$$
U^{-1} \mathscr{N} U=\mathscr{M}_{n}
$$

then for every strong neighbourhood $\Omega$ of $I$ in $\mathscr{R}$, there exists a $m^{\prime}>m, a$ unitary operator $U_{1}$ in $\mathscr{R}$ and $\mathscr{M} \subset \mathscr{M}_{m^{\prime}}$ with the following properties:

$$
\begin{aligned}
& \mathrm{i}-U_{1}^{-1} \mathscr{N}_{m} U_{1}=\mathscr{M} \\
& \text { ii }-U A U^{-1}=U_{1} A U_{1}^{-1} \quad \forall A \in \mathscr{M}_{n} \\
& \text { iii }-U_{1} U^{-1} \in \Omega . \\
& \text { Proof. Let us define } \mathscr{A}_{1}=U \mathscr{A} U^{-1} \text { and } \\
& \mathscr{A}_{2}=\overline{\mathscr{N}^{\prime} \cap \bigcup_{n} U \mathscr{M}_{n} U^{-1}} \\
& \mathscr{B}_{2}=\overline{\mathscr{N}^{\prime} \cap \bigcup_{n} \mathscr{N}_{n} .}
\end{aligned}
$$

One can see without difficulty that $\mathscr{A}_{2}$ and $\mathscr{B}_{2}$ have quasi-local structure and moreover that they both generate the same von Neumann algebra $\mathscr{N}^{\prime} \cap \mathscr{R}$ (cf. [2], Lemma 3) hence we can apply Lemma 4.3:

for every strong neighbourhood $\Omega$ of $I$ in $\mathscr{N}^{\prime} \cap \mathscr{R}$, there exists a $m^{\prime}>m$, a unitary operator $U_{11} \in \mathscr{N}^{\prime} \cap \mathscr{R}$ and a factor of type $I_{\infty}$

such that

$$
\mathscr{M}_{11} \subset \mathscr{N}^{\prime} \cap U \mathscr{M}_{m^{\prime}} U^{-1} \subset \mathscr{A}_{2}
$$

and $U_{11} \in \Omega$.

$$
U_{11}^{-1}\left(\mathscr{N}^{\prime} \cap \mathscr{N}_{m}\right) U_{11}=\mathscr{M}_{11}
$$


Let $\mathscr{M}$ be the von Neumann algebra generated by $\mathscr{N}$ and $\mathscr{M}_{11}$; then satisfies i).

$$
U_{1}=U_{11} U
$$

Moreover $U_{11}=U_{1} U^{-1} \in \Omega$, so iii).

On the other hand

$$
\mathscr{M}=U^{-1}\left(\mathscr{N} \cup \mathscr{M}_{11}\right)^{\prime \prime} U \subset \mathscr{M}_{m^{\prime}} .
$$

Finally if $A \in \mathscr{M}_{n}$, then

$$
U A U^{-1} \in \mathscr{N}
$$

since $U_{11} \in \mathscr{N}^{\prime}$, hence

$$
U_{1} A U_{1}^{-1}=U_{11} U A U^{-1} U_{11}^{-1}=U A U^{-1}
$$

which completes the proof.

Lemma 4.5. Let $\mathscr{A}=\bar{\bigcup}_{n} \mathscr{M}_{n}$ and $\mathscr{B}=\overline{\bigcup_{m} \mathscr{N}_{m}}$ be $C$-algebras with quasi-local structure acting on separable Hilbert spaces $H$ and generating the same factor $\mathscr{R}$ of type $I_{\infty}$; then there exists a unitary operator $U \in \mathscr{R}$ such that

$$
U \mathscr{A} U^{-1}=\mathscr{B} .
$$

Proof. Let $f_{i}$ be a countable set of vectors of the unit ball of $H$; then we construct by induction

$\mathrm{i}$ - a strictly increasing sequence $\mathscr{R}_{i}$ of factors of type $I_{\infty}$ in $\mathscr{A}$;

ii - a strictly increasing sequence $\mathscr{S}_{i}$ of factors of type $I_{\infty}$ in $\mathscr{B}$;

iii - a sequence of unitaries $U_{i}$ of $\mathscr{R}$ such that

iv $-U_{i} \mathscr{R}_{i} \mathrm{U}_{i}^{-1}=\mathscr{S}_{i}$

$\mathrm{v}-U_{i+1} A U_{i+1}^{-1}=U_{i} A U_{i}^{-1}$ if $A \in \mathscr{R}_{i}$

vi - $\left\|\left(U_{i+1}-U_{i}\right) f_{j}\right\|<2^{-i} \forall j \leqq i$

vii - if $i$ is odd, there exists $n_{i}$ such that

$$
\mathscr{R}_{i}=\mathscr{M}_{n_{i}}
$$

viii - if $j$ is even, there exists $m_{j}$ such that

$$
\mathscr{S}_{j}=n_{m_{j}} .
$$

Indeed, let $\mathscr{R}_{1}=\mathscr{M}_{1}$; assume $\mathscr{R}_{i}$ is given for all $i \leqq 2 k+1$ with $\mathscr{R}_{2 k+1}=\mathscr{M}_{n}$, $\mathscr{S}_{i}$ and $U_{i}$ for $i<2 k+1$; by Lemma 4.4 , there exists $m>n, U_{2 k+1}, \mathscr{S}_{2 k+1}$ such that

$$
\begin{gathered}
U_{2 k+1} \mathscr{R}_{2 k+1} U_{2 k+1}^{-1}=\mathscr{S}_{2 k+1} \\
U_{2 k+1} A U_{2 k+1}^{-1}=U_{2 k} A U_{2 k}^{-1} \quad \forall A \in \mathscr{R}_{2 k}
\end{gathered}
$$

moreover one can choose the strong neighbourhood

$$
\Omega=\left\{U_{2 k} f_{i} ; i \leqq 2 k ; \varepsilon=2^{-2 k}\right\}
$$


hence by Lemma 4.4:

and

$$
\left\|\left(U_{2 k+1} U_{2 k}^{-1}-\mathbb{1}\right) U_{2 k} f_{i}\right\|<2^{-2 k} \quad i \leqq 2 k
$$

Let $\mathscr{S}_{2 k+2}=\mathscr{N}_{m}$.

$$
\mathscr{S}_{2 k+1} \subsetneq \mathscr{N}_{m} .
$$

Using again Lemma 4.4, there exists $m^{\prime}>m, U_{2 k+2}, R_{2 k+2}$ such that

$$
\begin{gathered}
U_{2 k+2} \mathscr{R}_{2 k+2} U_{2 k+2}^{-1}=\mathscr{S}_{2 k+2} \\
U_{2 k+2} A U_{2 k+2}^{-1}=U_{2 k+1} A U_{2 k+1}^{-1} \quad \forall A \in \mathscr{R}_{2 k+1} \\
\left\|\left(U_{2 k+2}-U_{2 k+1}\right) f_{i}\right\|<2^{-(2 k+1)} \quad i \leqq 2 k+1 \\
\mathscr{R}_{2 k+2} \subsetneq \mathscr{M}_{m^{\prime}} .
\end{gathered}
$$

One can choose

$$
\mathscr{R}_{2 k+3}=\mathscr{M}_{m^{\prime}}
$$

which completes the construction.

By vi) the sequence of $U_{i}$ converges (cf. [15], p. 155). If $U=S \cdot \lim _{r \rightarrow \infty} U_{r}$ then $U \in \mathscr{R}$ and $U$ is an isometric mapping from $H$ to $H$.

Moreover the mapping

$$
\Phi(A)=U^{*} A U
$$

is an isomorphism of $\mathscr{B}$ into $\mathscr{A}$.

Indeed for any $r \geqq n U_{n}^{*} A U_{n}=U_{r}^{*} A U_{r} A \in \mathscr{S}_{n}$ and

so that

$$
W \cdot \lim _{r \rightarrow \infty} U_{r}^{*} A U_{r}=U^{*} A U
$$

and

$$
U^{*} A U=U_{n}^{*} A U_{n} \quad \forall A \in \mathscr{S}_{n}
$$

$$
U^{*} \mathscr{S}_{n} U=\mathscr{R}_{n} .
$$

Consequently $\Phi$ is an isomorphism of $\bigcup_{n} \mathscr{S}_{n}$ onto $\bigcup_{n} \mathscr{R}_{n}$ which are both dense respectively in $\mathscr{B}$ and $\mathscr{A} ; \Phi$ is clearly continuous hence it extends to an isomorphism of $\mathscr{B}$ onto $\mathscr{A}$.

Moreover an argument quite similar to that of Powers ([15], p. 156) shows that $U$ is unitary.

Theorem 4.6. Let $\mathscr{A}$ be a $C *$-algebra with a quasi-local structure; the group of *-automorphisms of $\mathscr{A}$ acts transitively within the pure locally normal states of $\mathscr{A}$.

Proof. Let $\omega_{1}$ and $\omega_{2}$ be two pure locally normal states of $\mathscr{A} . H_{i}, \pi_{i}, \Omega_{i}$ $(i=1,2)$ the representation space, the representation and the cyclic vector defined by $\omega_{i}$; since $H_{i}(i=1,2)$ are separable Hilbert spaces, we can identify them. 
It is clear that $\pi_{i}(\mathscr{A})$ fulfils conditions of Lemma 4.5 so that there exists a spatial $*$-isomorphism $\Phi$ of $\pi_{1}(\mathscr{A})$ onto $\pi_{2}(\mathscr{A})$; the representations $\pi_{i}$ are injective, hence there exists a $*$-automorphism $\alpha^{\prime}$ of $\mathscr{A}$ such that

$$
\Phi \circ \pi_{1}=\pi_{2} \circ \alpha^{\prime} .
$$

The states $\omega_{1}$ and $\omega_{2} \circ \alpha^{\prime}$ define unitarily equivalent irreducible representations of $\mathscr{A}$ and consequently there exists an automorphism $\alpha^{\prime \prime}$ of $\mathscr{A}$ such that

$$
\omega_{1}=\omega_{2} \circ \alpha^{\prime} \circ \alpha^{\prime \prime}=\omega_{2} \circ \alpha .
$$

We are indebted to Professor H. Borchers for the following remark: Lemma 4.2 can be generalized to the case where $\mathscr{R}$ is an arbitrary von Neumann algebra.

Let us sketch the proof of this fact: with the same notations as previously, take $E_{n}$ (resp. $F_{n}$ ) a sequence of orthogonal minimal projections of sum $\mathbb{1}$ in $\mathscr{R}_{1}$ (resp. in $\mathscr{R}_{2}$ ). $E_{1}$ and $F_{1}$ are properly infinite projections, with central carrier $\mathbb{1}$ with respect to $\mathscr{R}$, so that they are equivalent ([14], chap. III, $\S 8$, cor. 5 and Chap. III, $\S 1$, prop. 1). Let $V$ isometric in $\mathscr{R}$ be such that

$$
V V^{*}=E_{1} \quad V^{*} V=F_{1} .
$$

Defining the sequence of partially isometric $V_{n}$ (resp. $\left.W_{n}\right)$ in $\mathscr{R}_{1}$ (resp. $\mathscr{R}_{2}$ ) such that

one can choose

$$
\begin{array}{ll}
V_{n} V_{n}^{*}=E_{n} & \left(\text { resp. } W_{n} W_{n}^{*}=F_{n}\right) \\
V_{n}^{*} V_{n}=E_{1} & \left(\text { resp. } W_{n}^{*} W_{n}=F_{1}\right)
\end{array}
$$

$$
U=\sum_{n} W_{n} V^{*} V_{n}^{*} \in \mathscr{R}
$$

hence the result follows.

The Lemmas 4.3 to 4.5 can be extended to $\mathscr{R}$ an arbitrary von Neumann algebra so that one can state the more general result:

Theorem 4.7. Let $\pi_{1}$ and $\pi_{2}$ be two locally normal representations of a $C^{*}$-algebra $\mathscr{A}$ with a quasi-local structure.

They generate isomorphic von Neumann algebras iff there exists a *-automorphism $\alpha$ of $\mathscr{A}$ such that $\pi_{1}$ and $\pi_{2} \circ \alpha$ are quasi-equivalent.

The proof is standard, cf. [15], p. 157.

Acknowledgments. It is a pleasure to thank Professor D. Kastler for his interest in our work. We are also indebted to H. Borchers, S. Doplicher, A. Grossmann and M. Winnink for fruitful discussions. 


\section{References}

1. Winnink, M.: Thesis, Groningen (1968).

2. Haag, R., Kadison, R. V., Kastler, D.: Commun. math. Phys. 16, 81 (1970).

3. Sakai, S.: The Theory of $W^{*}$-algebras. Mineographied notes, Yale University (1962).

4. Hugenholtz, N.M., Takesaki, M., Winnink, M.: Local Normality of the K.M.S. states in Quantum Statistical Mechanics, in preparation.

5. Haag, R., Hugenholtz, N. M., Winnink, M.: Commun. math. Phys. 5, 215 (1967).

6. Schwartz,L.: Théorie des distributions. Herman: Paris 1966.

7. Araki,H.: Progr. Theor. Phys. 32, 844 (1964).

8. Doplicher, S., Kastler, D., Størmer, E.: J. Functional Analysis 3, 419 (1969).

9. Rocca, F., Sirugue, M., Testard,D.: Commun. math. Phys. 13, 317 (1969).

10. Rocca, F., Sirugue, M., Testard,D.: Commun. math. Phys. 19, 119 (1970).

11. Powers, R. T., Størmer, E.: Commun. math. Phys. 16, 1 (1970).

12. Manuceau, J.: Ann. Inst. Henri Poincaré 2, 139 (1968).

13. Manuceau, J., Verbeure, A.: Commun. math. Phys. 9, 293 (1968).

14. Dixmier, J.: Les algébres d'opérateurs dans l'espace hilbertien. Paris: Gauthier-Villars 1968.

15. Powers, R. T.: Ann. Math. 86, 138 (1967).

16. Ruelle, D.: Commun. math. Phys. 11, 339 (1969).

Dr. M. Sirugue

Centre de Physique Théorique - C.N.R.S.

31, chemin J. Aiguier

F-13 Marseille $9^{\circ}$, France 